\title{
Schwerpunkt Gerontopsychiatrie im Fachgebiet Psychiatrie und Psychotherapie - Kontra
}

\author{
Geriatric Psychiatry as a Subspecialty in the Field of Psychiatry and \\ Psychotherapy - Contra
}

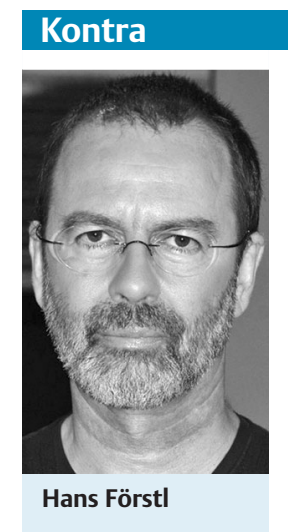

Hans Förstl

Deutschland ist auch heute dank seiner langen Duodezfürstentradition eine mitteleuropäische Region mit einem starken Hang seiner Bürger zu Separatismus und Klüngelwirtschaft, ein Land der Vereinsmeier von der Kaninchenzüchtung bis zur Psy-

chosomatik. Erfolgreich vom Korpus der Seelenheilkunde abgetrennt wurde auch bereits die Kinder- und Jugendpsychiatrie. Gleiches soll nun am anderen Ende mit der Gerontopsychiatrie geschehen und dies käme einerseits den Interessen weniger Gerontopsychiatriephiler entgegen, andererseits schlichteren allgemeinpsychiatrischen Gemütern, die sich nun allein der Dementia praecox hingeben könnten.

\section{Die Psychiatrie kann nicht auf die Gerontopsychiatrie verzichten}

Was bliebe der Kernpsychiatrie denn noch übrig? Leichtere affektive Erkrankungen werden heute je nach Neigung der Patienten medikamentös von Ärzten aller Fachrichtungen oder von psychologischen Psychotherapeuten versorgt. Der Berufsstand Psychosomatik schickt sich kregel an, alle weniger schweren Störungen aus dem Umkreis der Medizin - sogar der Psychiatrie - aufzusammeln, deren Betroffene ausreichende Begeisterung für längerfristige Gespräche mitbringen, solange die Kassen dies finanzieren. Sozialpädagogen - die in diesem Zusammenhang viel zu selten genannte und dabei ganz wesentliche Kraft - sind imstande, längerfristig Kranken ein vernünftiges Auskommen zu sichern. Betroffenenverbände betonen gelegentlich das Recht auf Krankheit und signalisieren zur Verzweiflung der Angehörigen, dass sich Psychiater und andere gefälligst heraushalten sollen. Künstler, Journalisten und Politiker ge- stalten das dann auch noch ganz gerne ästhetisch, kritisch und demokratisch aus. Also ein klares Signal an den Allgemeinpsychiater, sich nicht allzu stark zu engagieren. Sollte von Staats wegen eines Tages der Ruf nach einer Art von psychischer Gesundheitspolizei laut werden, sollten Psychiater Zurückhaltung üben und getrost abwarten wer sich meldet. Sowohl Polizisten als auch Sonderpädagogen wären in zweiwöchigen Abendkursen entsprechende Kenntnisse verbindlich zu vermitteln.

Damit drohte ganz am Ende sogar die mächtige Gerontopsychiatrie eine schmächtige Schizophrenologie zu überragen, denn das Alter ist groß im Kommen. Die Lebenserwartung steigt - optimistischen Schätzungen entsprechend um 3 Monate pro Jahr. Zugrunde liegt vor allem eine immer länger währende Lebensphase bei immer besserer Gesundheit an Körper und Geist. Dies ist auch ein Erfolg für die gesamte Medizin und gleichzeitig eine Verpflichtung zu lebenslanger Gesundheitserziehung vom Neugeborenen bis zum Greis und zu immer konsequenterer Behandlung der medizinischen Probleme aller Lebensalter. Psychiater sollten eine Beteiligung an diesen Maßnahmen nicht aus falsch verstandenem Spezialistentum zurückweisen; auch die frühzeitige Erkennung und Behandlung von affektiven, psychotischen und vielen anderen Gefährdungen verbessert langfristig Lebenserwartung und Lebensqualität. Und dennoch werden die Menschen zwangsläufig in hohen Jahren immer häufiger von psychischen Störungen eingeholt, die als Folgen einer veränderten sozialen oder organischen Situation verstanden werden können. Mangelnde Sozialkontakte, eine verminderte Leistungsfähigkeit von Gehirn und Restorganismus verändern die kognitive und emotionale Verfassung.

Mors certa, hora incerta ist eine dieser klassischen Binsenweisheiten, die im Kontext der altersassoziierten Erkrankungen aktuell an Brisanz gewinnt. So wäre bei Betrachtung der epidemiologischen Daten zu paraphrasieren Dementia certa, hora incerta. Nur der vorzeitige Tod von zwei Dritteln der Menschen an anderen Erkrankungen verhindert derzeit, dass noch mehr als ein Drittel eine Demenz erlebt. Aber auch $30 \%$ sind ein stattlicher Prozentsatz. Zumal leichtere kognitive Defizite und die Vorboten der Demenz hierbei nicht eingeschlossen sind. Partielle kognitive Einschränkungen können eigenwilligen paranoiden Entwicklungen Vorschub leisten, denen man bei jüngeren Patienten kaum begegnet und sie werfen doch ein Licht auf die verschrobene, egozentrische Arbeitsweise unseres Gehirns, die bei voller intellektueller Leistungsfähigkeit über lange Strecken geschickt kaschiert wird. Die Depression im Alter wurde lange Zeit und wird auch heute noch von manchen als selbstverständlicher und eigentlich alterstypischer Normalzustand hingenommen. Auch bei eindeutigen und schwerwiegenden Anlässen zur Trauer sollte nicht so lange mit einer Therapie gezögert werden bis sich die Affektlage verfestigt hat und das noch vorhandene Umfeld durch einen störrischen Alten so vor den Kopf gestoßen wurde bis es sich endgültig verabschiedet hat.

Verwirrtheitszustände sind der Lackmustest eines funktionierenden Gesundheitssystems. Die Versorgung älterer, multimorbider - v.a. zerebral multimorbider und polypharmazierter Menschen in Praxis und Klinik sind in der Bundesrepublik in einem jämmerlichen Zustand. Auch wenn die Worte multi- und interdisziplinär allergisierend wirken, so sind sie hier angebracht. Im Gegensatz zu anderen entwickelten Ländern fallen die verwirrten Alten bei uns durch das Raster der meisten modernen Krankenhäuser. Sie oder ihre Angehörigen müssen selber sehen wo sie bleiben. Sitzwachen gibt es kaum, der Psychiater wird schnell notfallmäßig konsultiert und empfiehlt Neuroleptika sowie Fünfpunktfixierung, Geriater gibt es nicht und schon gar keinen proaktiven Plan zur Risikoreduktion und raschen Re- 
kompensation. Auch dies ist eine allgemeinpsychiatrische Aufgabe und derzeit kein Thema, das von einem versprengten gerontopsychiatrischen Expertengrüppchen gestemmt werden könnte.

Praktische Ärzte und Allgemeinmediziner sind meist die zuverlässigeren und besseren Behandler alter Patienten mit typischen gerontopsychiatrischen Problemen als jene Psychiater und Psychotherapeuten, die keine Zeit und wenig Ahnung haben. Sie sind in ihren Praxen entweder seit Jahrzehnten als Spezialisten für Schizophrenie intellektuell eingeengt oder seit ihrer Niederlassung als praktizierende Seelentröster auf einen 55-MinutenRhythmus geeicht, der keine Abweichung mehr zulässt. Dieser flagrante Facettenreichtum innerhalb der Psychiatrie würde zweifelsfrei durch eine weitere Kruste knusprig bereichert. Wollte man die Schwächen des Faches also noch weiter ausbauen, wäre eine gesonderte Schwerpunktsetzung auf Gerontopsychiatrie und -psychotherapie unbedingt zu unterstützen. Vonseiten der Neurologen und der Geriater ist auch keine Hilfe für die Patienten zu erwarten und den Psychiatern droht nicht die Gefahr einer kompetenten und gewichtigen Konkurrenz; die einen kümmern sich mehr um Krankheiten als um Menschen und die anderen zählen nicht (es sind einfach zu wenige).

Während sich die Forschung in einigen anderen Bereichen der Psychiatrie im Kreise dreht beziehungsweise in Nachbardisziplinen ausgelagert wird, erwies sich das bis vor 20 Jahren vernachlässigte und bisweilen sogar verachtete Thema Gerontopsychiatrie in enger Kooperation mit Epidemiologie, Bildgebung und Molekularbiologie als besonders fruchtbar. Hier wurden Erkrankungen grundsätzlich neu verstanden und es zeichnen sich Perspektiven innovativer und kausaler Interventionen ab, die wesentlich klarer konturiert sind als in den anderen Bereichen des Faches. Dass der Psychiatrie in der Bundesbananenrepublik das Thema Demenz zugunsten neurodegenerativer Erkrankungen durch einen dynastischen Drahtzieher handstreichartig entwunden werden konnte, belegt auch, um wie viel stärker Gerontopsychiatrisches in der Psychiatrie verankert werden muss.
Ceterum censeo: Die Diagnostik und Therapie alter Menschen mit psychischen Erkrankungen ist ein genuiner, ein essenzieller, das heißt ganz und gar unverzichtbarer Bestandteil der Psychiatrie. Jeder Psychiater muss Gerontopsychiatrie können. Sie ist das Thema Nummer 1 einer künftigen Psychiatrie und Psychotherapie. Die Schaffung einer Neigungsgruppe Alterspsychiatrie ist daher nicht nur nicht notwendig, sondern sogar vollkommen überflüssig. Gerontopsychiatrie hat nichts, was die Psychiatrie nicht hat (und braucht).

\section{Korrespondenzadresse}

Prof. Dr. Hans Förstl

TU München, Klinikum rechts der Isar Ismaninger Str. 22

81675 München

hans.foerst|@|rz.tu-muenchen.de

\section{Bibliografie}

Dol http://dx.doi.org/

10.1055/s-0033-1349540

Psychiat Prax 2013; 40: 370-371

(c) Georg Thieme Verlag KG

Stuttgart · New York

ISSN 0303-4259 